\title{
Transient residence and exposure times
}

\author{
E. J. M. Delhez \\ University of Liège, Mathematical Methods and Modelling, Belgium \\ Received: 11 April 2005 - Published in Ocean Science Discussions: 26 May 2005 \\ Revised: 1 December 2005 - Accepted: 6 December 2005 - Published: 9 January 2006
}

\begin{abstract}
The residence time measures the time spent by a water parcel or a pollutant in a given water body and is therefore widely used in environmental studies. The adjoint method introduced by Delhez et al. (2004) to compute this diagnostic is revised here to take into account the effect of the initialization and of the boundary conditions.

In addition to the equation for the mean residence time, it is suggested to solve a simple advection-diffusion problem to quantify the effect of the initialization and clarify the interpretation of the results.

Using the two same equations but with modified boundary conditions, the method can also be used to quantify the accumulated time spent by water/tracer parcels in a control domain. This diagnostic is called "exposure time".

Analytical and realistic model results are used to illustrate the concepts.
\end{abstract}

\section{Introduction}

The residence time of a water parcel in a water body is usually defined as the time taken by this parcel to leave this water body (e.g. Bolin and Rhode, 1973; Takeoka, 1984; Zimmerman, 1988; Monsen et al., 2003; Braunschweig et al., 2003). As such, it is a valuable diagnostic tool to describe and understand environmental issues. The residence time provides indeed a global measure of the influence of the hydrodynamic processes on the aquatic systems. In environmental studies, this time scale can be compared with characteristic biochemical activity rates to understand the dynamics of a system (e.g. Nixon et al., 1996; Braunschweig et al., 2003; Hydes et al., 2004) or assess the vulnerability of a water domain to potential pollution and eutrophication problems (e.g. Vollenweider, 1976). In a different context, the residence time can

Correspondence to: E. J. M. Delhez

(e.delhez@ulg.ac.be) be used as a measure of the time spent by eggs or larvae in a suitable habitat (e.g Wang et al., 2003; Harley et al., 2004).

Basically, the residence time is a property of each water parcel ; it is Lagrangian by nature. Indeed, the straightforward procedure to assess the residence time consists in injecting some tracer in the flow, following the path of these tracer parcels and registering the time when they leave the domain of interest. This procedure can be equally applied in real world experiments or in numerical model simulations.

Mathematically, the mean residence time $\bar{\theta}(t, \mathrm{x})$ at time $t$ and location $\mathrm{x}$ can be computed by monitoring the temporal evolution $\tilde{m}_{(t, \mathrm{x})}(t+\tau)$ of the mass of the tracer in the control region at the time $t+\tau$ after a unit point release at $(t, \mathrm{x})$. Following Bolin and Rhode (1973) and Takeoka (1984), one can write

$\bar{\theta}(t, \mathrm{x})=\int_{0}^{1} \tau d \tilde{m}_{(t, \mathrm{x})}$

Delhez et al. (2004) introduced an alternative procedure designed for numerical models. They showed that the residence time can be computed as the solution of an advectiondiffusion problem with a unit source term and appropriate boundary conditions. The method provides the variations in space and time of the residence time with a single model run. The method doesn't require any Lagrangian module. It is Eulerian by nature which makes it more appropriate to longterm and large scale simulations than the straightforward Lagrangian approach. Considering the potential discrepancies between the Lagrangian and Eulerian descriptions of diffusion, the Eulerian approach of the residence time is closer to the Eulerian hydrodynamic models and represents a more direct diagnostic of the model results.

In their paper, Delhez et al. (2004) raise the issue of the appropriate definition of the residence time when water parcels leaving the domain of interest are allowed to re-enter at later times. They also mention the problem associated with the fact that a finite range simulation cannot provide the 
residence time of all the water parcels nor the mean residence time.

The purpose of this paper is to clarify these two issues and complement the adjoint method advocated by Delhez et al. (2004) with appropriate definitions and additional control variables.

\section{Backward procedure for the computation of the resi- dence time}

Because of diffusion, different water parcels released at the same location and the same time follow different paths, exit the control domain $\omega$ at different times and have therefore different residence times in $\omega$. To describe this situation, Delhez et al. (2004) define the cumulative distribution function $D(t, \tau, \mathrm{x})$ as the fraction of the mass of the tracer released at time $t$ and location $\mathrm{X}$ whose residence time is larger or equal to $\tau$. This is also the mass of tracer in the control region at time $t+\tau$ following a unit release at time $t$ and location $\mathrm{x}$, therefore

$D(t, \tau, \mathrm{x})=\tilde{m}_{(t, \mathrm{x})}(t+\tau)$

This cumulative distribution function is shown to satisfy

$$
\left\{\begin{array}{l}
\frac{\partial D}{\partial t}-\frac{\partial D}{\partial \tau}+\boldsymbol{v} \cdot \nabla D+\nabla \cdot[\boldsymbol{K} \cdot \nabla D]=0 \\
D(t, 0, \mathbf{x})=\delta_{\omega}(\mathbf{x})
\end{array}\right.
$$

where $\boldsymbol{v}$ is the velocity vector, $\boldsymbol{K}$ denotes the symmetric diffusion tensor and

$\delta_{\omega}(x)=\left\{\begin{array}{lll}1 & \text { if } & x \in \omega \\ 0 & \text { if } & x \notin \omega\end{array}\right.$

is the characteristic function of the control region $\omega$.

The zeroth order moment of the cumulative distribution function, i.e.

$\bar{\theta}(t, \mathrm{x})=\int_{0}^{\infty} D(t, \tau, \mathrm{x}) d \tau$

is the mean residence time if $D$ satisfies particular boundary conditions (Delhez et al., 2004).

Assuming that $D(t, \tau, \mathrm{x})$ decreases to zero when $\tau$ tends to infinity, i.e. that the whole material is eventually flushed out of the control region, Eq. (3) can be integrated with respect to $\tau$ to simplify the problem into the more classical differential problem

$\frac{\partial \bar{\theta}}{\partial t}+\delta_{\omega}+\boldsymbol{v} \cdot \nabla \bar{\theta}+\nabla \cdot[\boldsymbol{K} \cdot \nabla \bar{\theta}]=0$

for $\bar{\theta}(t, \mathrm{x})$. For $\bar{\theta}$ to be equal to the mean residence time, Eq. (6) must be solved with the boundary condition that $\bar{\theta}$ vanishes on the boundary $\delta \omega$ of the control domain.

Equations (3) and (5) are derived from the adjoint of the forward advection-diffusion problem. They must therefore be integrated backwards in time with homogeneous boundary conditions. The integration backwards in time is clearly necessary for stability reasons associated with the apparent negative diffusion term in Eqs. (3) and (6). It is also a consequence of the fact that one does not know in advance the fate of the particles.

\section{Finite range simulation}

In principle, Eqs. (3) and (6) must be integrated backwards from $t=+\infty$ in order to be able to describe the full distribution of residence times, including the fate of particles with a very large residence time. In practice, of course, the equation is integrated backwards from some finite time $T$ at which the real conditions are unknown. As a result, the solution will not provide the exact mean residence time until the uncertainty about the initial conditions has disappeared. Intuitively, one can expect that the effect of the initial conditions smears out after a period of integration of several multiples of the residence time.

A more accurate appraisal of the effect of the initial conditions can be given by a careful analysis of Eq. (3). Clearly, the uncertainty about what happens after the "initial" time $T$ affects only the cumulative distribution $D(t, \tau, \mathrm{x})$ in the range of $\tau>T-t$. As $t$ decreases, while proceeding with the backward integration, an increasing portion of the distribution of the residence time is uncovered.

If the initial condition at time $T$ is $D=0$, then $D$ is also zero for all $\tau>T-t$,

$\int_{0}^{\infty} D(t, \tau, \mathrm{x}) d \tau=\int_{0}^{T-t} D(t, \tau, \mathrm{x}) d \tau$

and the solution $\bar{\theta}$ of Eq. (6) characterizes only the water parcels with a residence time smaller than $T-t$. While $\bar{\theta}$ tends to the mean residence time for large values of $T-t$, the actual rate of convergence is not known.

To quantify the proportion of water parcels whose contribution is taken into account in $\bar{\theta}$, we propose to solve the adjoint problem

$\left\{\begin{array}{l}\frac{\partial C_{T}^{*}}{\partial t}+\boldsymbol{v} \cdot \nabla C_{T}^{*}+\nabla \cdot\left[\boldsymbol{K} \cdot \nabla C_{T}^{*}\right]=0 \\ C_{T}^{*}(T, \mathrm{x})=\delta_{\omega}(\mathrm{x})\end{array}\right.$

in addition to Eq. (6). After Delhez et al. (2004), the solution $C_{T}^{*}(t, \mathrm{x})$ of this problem can indeed be interpreted as the proportion of the initial point release at $(t, \mathrm{x})$ that is still present in the control domain at time $T$. Conversely,

$\tilde{C}_{T}^{*}(t, \mathrm{x}) \equiv 1-C_{T}^{*}(t, \mathrm{x})$

represents the proportion of water parcels whose residence time can be computed with a model run in the time window $[t, T]$. This quantity can be used to quantify the representativeness of the solution of Eq. (6) as the mean residence 
time. Therefore, the scalar field with concentration $C_{T}^{*}(t, \mathrm{x})$ is called the "control scalar" in this paper.

To clarify the concepts introduced in this section, it is interesting to consider the highly idealized system of a onedimensional domain $x \in(-\infty, \infty)$ and compute the mean residence time in the control domain $\omega=(-\infty, 0]$. We assume that the velocity field is uniform but varies with time as in Fig. 1. Diffusion is first neglected to ease the understanding.

From the discussion above, the residence time is obtained as the solution $\bar{\theta}$ of

$$
\left\{\begin{array}{lrl}
\frac{\partial \bar{\theta}}{\partial t}+u(t) \frac{\partial \bar{\theta}}{\partial x}+1=0, & x \in \omega \\
\bar{\theta}(t, 0)=0 \quad \text { when } & u(t)>0
\end{array}\right.
$$

This equation must be integrated backwards from some "initial" time $T$ taken here as $T=3 \mathcal{T}$ (Cf. Fig. 1). As the true "initial" conditions at that time are unknown we take

$\bar{\theta}(T, x)=0$

The solution of the problem Eqs. (10-11) at different times is shown in Fig. 2.

At time $t=2 \mathcal{T}$, the residence time varies linearly between $\mathcal{T}$ at $x=-U \mathcal{T}$ and 0 at $x=0$. This is precisely the time for a water parcel to be advected from its location at $t=2 \mathcal{T}$ to the boundary of the control domain by the velocity field $U$ acting between $t=2 \mathcal{T}$ and $t=3 \mathcal{T}$.

The residence time for $x<-U \mathcal{T}$ seems to be a constant, equal to the elapsed time $\mathcal{T}$ of the backward simulation. This is however an artefact of the initialization of the computation at time $T$. The water parcels released at $x<-U \mathcal{T}$ at time $t=2 \mathcal{T}$ do not have time enough to exit the control domain; they are still in $\omega$ at $t=T$. Therefore the residence time of these water parcels cannot be settled since their exit time is unknown.

The resolution of the appropriate form of Eq. (8),

$$
\left\{\begin{array}{l}
\frac{\partial C_{T}^{\star}}{\partial t}+u(t) \frac{\partial C_{T}^{\star}}{\partial x}=0, \quad x \in \omega \\
C_{T}^{\star}(T, x)=1, \quad x \in \omega \\
C_{T}^{\star}(t, 0)=0 \quad \text { when } \quad u(t)>0
\end{array}\right.
$$

is useful to identify the part of the solution of Eq. (10) that is affected by the initialization and/or cannot be interpreted as the residence time. The solution $C_{T}^{\star}$ plotted in Fig. 2 confirms that the particles located at $x<-U \mathcal{T}$ at time $t=2 \mathcal{T}$ do not leave the domain during the simulation period.

A quick look at the distribution of the control scalar at $t=\mathcal{T}$, tells us that none of the particles present in the control domain at this time can leave the control domain before $t=T$. Their residence time is therefore unknown. The value of $\bar{\theta}$ is just a lower bound of the residence time.

A similar analysis can be done from the results at $t=0$. In this case, the residence time increases linearly from zero at

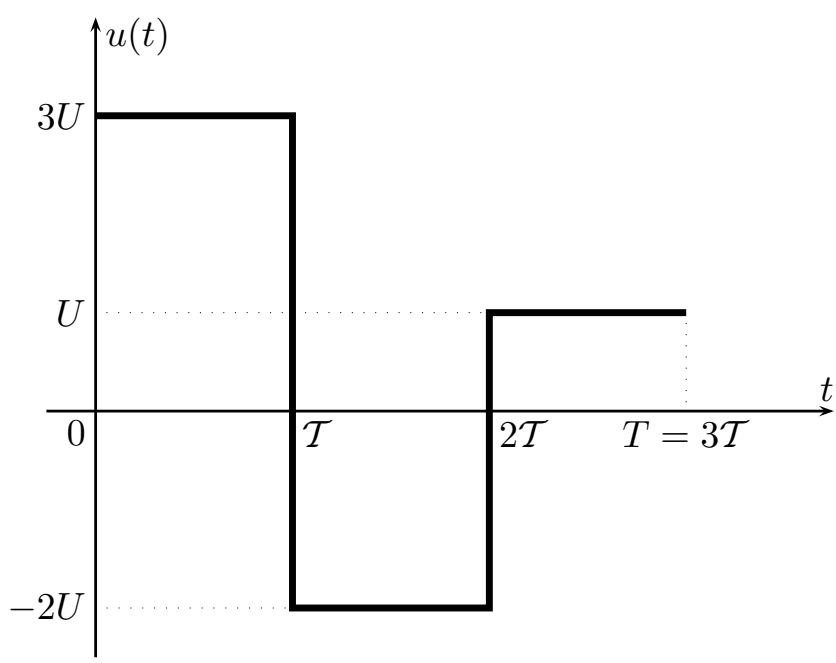

Fig. 1. Temporal evolution of the velocity $u(t)$.

the origin to $\mathcal{T}$ at $x=-3 U \mathcal{T}$. The residence time cannot be computed in the leftmost part of the control domain.

Similar results are obtained if some diffusion is added to the dynamics (Fig. 2). In this case, one must solve

$\left\{\begin{array}{l}\frac{\partial \bar{\theta}}{\partial t}+u(t) \frac{\partial \bar{\theta}}{\partial x}+\kappa \frac{\partial^{2} \bar{\theta}}{\partial x^{2}}=0, \quad x \in \omega \\ \bar{\theta}(T, x)=0, \quad x \in \omega \\ \bar{\theta}(t, 0)=0\end{array}\right.$

and

$\left\{\begin{array}{l}\frac{\partial C_{T}^{\star}}{\partial t}+u(t) \frac{\partial C_{T}^{\star}}{\partial x}+\kappa \frac{\partial^{2} \bar{C}_{T}^{\star}}{\partial x^{2}}=0, \quad x \in \omega \\ C_{T}^{\star}(T, x)=1, \quad x \in \omega \\ C_{T}^{\star}(t, 0)=0\end{array}\right.$

by backward integration from $t=T$. Similar conclusions are obtained with, of course, smoother spatial distributions of the residence time and of the control scalar $C_{T}^{\star}$. Because of diffusion, the initialization is seen to affect the results in a larger part of the control domain and/or for earlier times $t$. The spatial distribution of the control scalar is not strictly equal to unity in the control domain, even in its leftmost part. This shows that some water parcel can now escape the control domain by diffusion within the studied time window. In such areas, $\bar{\theta}$ provides a lower bound for the residence time but cannot be interpreted as a valid approximation of the residence time unless $C_{T}^{\star}$ is close to zero.

\section{A priori estimate of the initialization time}

The computation of $C_{T}^{*}$ is useful to check the influence of the initialization a posteriori, i.e. once the simulation has been 

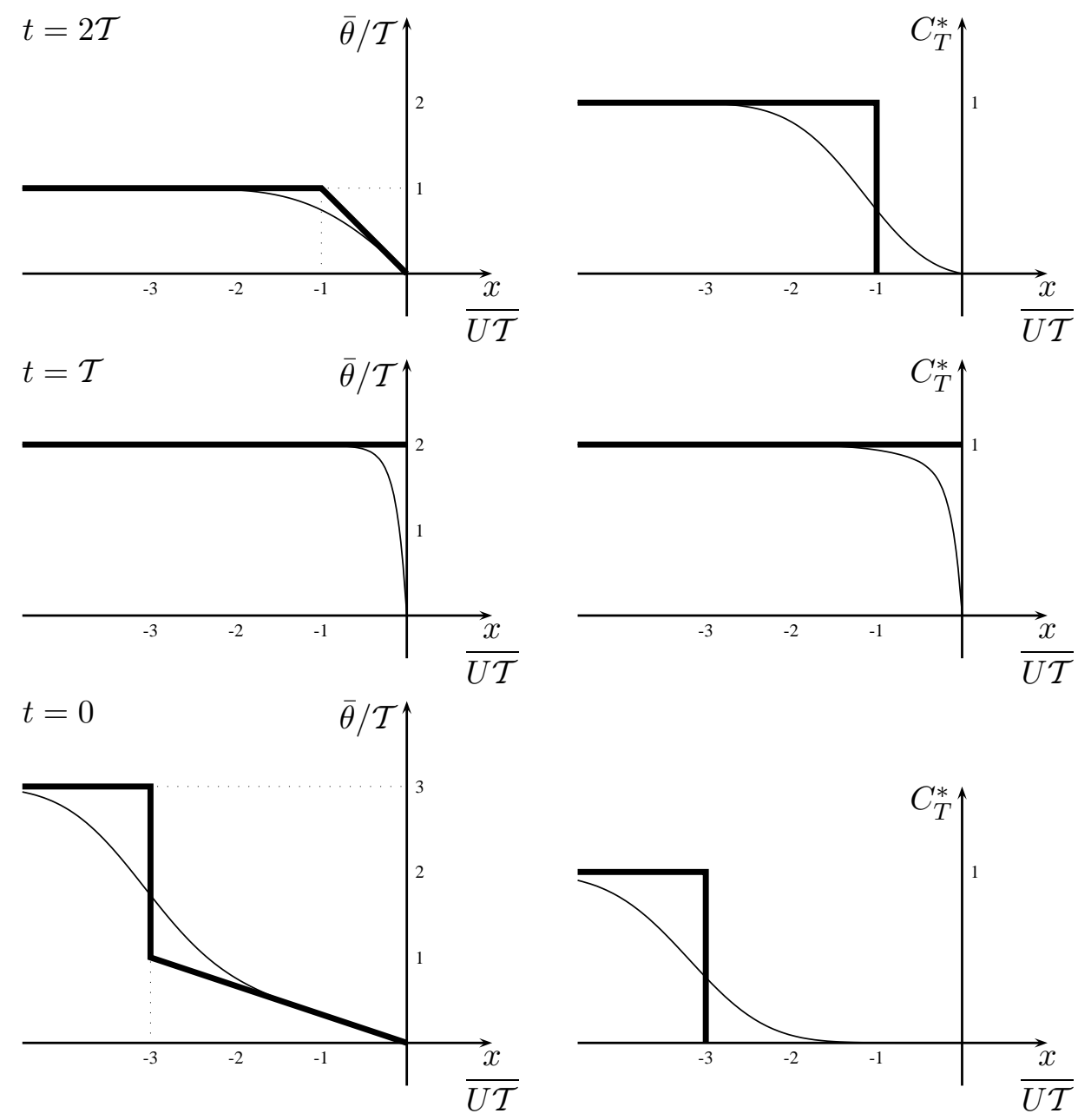

Fig. 2. Temporal evolution of $\bar{\theta}$ and $C_{T}^{*}$ from a backward integration of the equations for the mean residence time from $t=T$. Results without diffusion (thick curve) and with diffusion (thin curve, $\kappa=U^{2} \mathcal{T} / 4$ ).

carried out. For practical applications, it is also desirable to have some a priori estimates of the spin-up time. A compromise must indeed be found between the necessity to take the "initial time" $T$ as large as possible to remove the effect of the initialization and the wish to reduce the length of the numerical simulations. Obviously, the ideal duration of the simulation depends on the residence time it-self; the larger the residence time, the larger the duration of the simulation. As a rule of thumb, one could argue that the simulation window $[t, T]$ should be as large as twice the mean residence time for the results at time $t$ to be significant. As the residence time is not known a priori, rough estimates based on simplified models can be used to choose $T$.

In an advection dominated flow, the backward integration of Eq. (8) produces a front generated at the boundary of the control domain and moving into it. If $U^{c}$ denotes the characteristic velocity of the flow and if the model is allowed to spin-up for $\Delta t$, then the space swept by the front during that time interval can be characterized by the advective length scale $U^{c} \Delta t$. In the meantime, horizontal diffusion smears out the front over a length scale which is some multiple $\alpha$, say $\alpha=3$, of the diffusion length scale $\sqrt{K^{c} \Delta t}$ where $K^{c}$ is some characteristic (explicit and implicit) horizontal diffusion coefficient. This spreading reduces the influence of the boundary signal in the interior of the control domain. Therefore, $C_{T}^{*}$ will be close to zero only at locations whose distance to the outflow boundary of the control domain is less than

$\mathcal{L}^{c}=U^{c} \Delta t-3 \sqrt{K^{c} \Delta t}$

At such locations, the residence time can be reasonably obtained from the solution of Eq. (6) after a spin-up time of $\Delta t$. If $L$ denotes the horizontal dimension of the whole control domain, then the model should be allowed to spin-up for $\Delta t$ such that

$L \leq \mathcal{L}^{c}=U^{c} \Delta t-3 \sqrt{K^{c} \Delta t}$ 
The estimate (15) applies reasonably well to the 1-D case discussed above if the reversal of the flow (Fig. 1) is properly taken into account. For instance, considering the initialization at $t=T$ and looking at the results at $t=2 \mathcal{T}$, Eq. (15) gives (taking $U^{c}=3 U$ and $K^{c}=U^{2} \mathcal{T} / 4$ )

$\mathcal{L}^{c}=-1.5 U \mathcal{T}$

which confirms that the residence time computed by Eq. (6) cannot be considered to be significant at any point of the control domain (Fig. 2). For the conditions prevailing for $t \in[0, \mathcal{T}]\left(U^{c}=3 U\right.$ and $\left.K^{c}=U^{2} \mathcal{T} / 4\right)$ Eq. (15) predicts that the initialization at time $t=\mathcal{T}$ would produce reasonable estimates of the residence time at $t=0$ for

$x>1.5 U \mathcal{T}$

which can be confirmed by inspection of Fig. 2 .

\section{Residence time vs. age theory}

The two Eqs. (8) and (6) are very similar to the two equation system introduced by Delhez et al. (1999) to compute the age of tracers. In the case of a conservative tracer, these can be written as

$\frac{\partial C}{\partial t}+\boldsymbol{v} \cdot \nabla C=\nabla \cdot(\boldsymbol{K} \cdot \nabla C)$

and

$\frac{\partial \alpha}{\partial t}+\boldsymbol{v} \cdot \nabla \alpha=C+\nabla \cdot(\boldsymbol{K} \cdot \nabla \alpha)$

where $C$ is the concentration of the tracer and $\alpha$ is the socalled age concentration. The mean age $\bar{a}$ is related to $C$ and $\alpha$ by

$\bar{a}=\frac{\alpha}{C}$

The method discussed here for the computation of the residence time can therefore be understood as an extension of the Constituent-oriented Age Theory (Delhez et al., 1999; Deleersnijder et al., 2001; Delhez and Deleersnijder, 2002). This consolidated theory is hence called "Constituentoriented Age and Residence time Theory" (CART).

In the system (19)-(20), $C$ measures the concentration of the tracer that, taking into account the effect of initialization, contributes to the age concentration. It plays therefore a similar part as the control scalar $C_{T}^{*}$ in the computation of the residence time. The age concentration $\alpha$ accumulates the contribution to the mean age of the different tracer parcels forming $C$. It is comparable to $\bar{\theta}$.

From this similarity of the concepts, it is tempting to modify the definition of the mean residence time according to

$$
\frac{\bar{\theta}}{\tilde{C}_{T}^{*}}
$$

as only the water parcels forming $\tilde{C}_{T}^{*}$ are taken into account in $\bar{\theta}$. This ratio would be interpreted as the mean residence time of the water parcels in $\tilde{C}_{T}^{*}$. However, this approach is not appropriate. The residence time is a Lagrangian property inasmuch as it can be computed for each and every water parcel by attaching a "virtual clock" to each parcel and recording its exit time from the control domain. But the path of a single virtual water parcel subjected to Fickian diffusion does not make sense in its own. The paths of different parcels forming a given patch are not independent from each other. This is best demonstrated by the contradiction which arises if one selects the parcels accounting for $\tilde{C}_{T}^{*}\left(t_{0}, \mathrm{x}\right)=1-C_{T}^{*}\left(t_{0}, \mathrm{x}\right)$ at some initial time $t_{0}<T$ and use this as initial conditions of a forward simulation; while the definition of $\tilde{C}_{T}^{*}$ implies that it vanishes in the control domain at time $T$, the forward simulation will produce a non zero distribution at that time. The particles accounting for $\tilde{C}_{T}^{*}\left(t_{0}, \mathrm{x}\right)$ all manage to escape the control domain only because other particles immersed in the same diffusive environment remain in $\omega$. With the Fickian model of diffusion, it is impossible to separate the fate of the particles that leave the control domain within a given time window and those that do not. The arguments leading to Eq. (22) are therefore inappropriate.

\section{Residence time and exposure time}

The physical interpretation of $\bar{\theta}$ as the mean residence time in the control domain $\omega$ depends on the boundary conditions used to solve Eqs. (3) or (6).

The residence time is usually defined as the time taken for a water parcel to leave the control domain for the first time (e.g. Bolin and Rhode, 1973; Takeoka, 1984; Zimmerman, 1988; Monsen et al., 2003). To compute this diagnostic, called strict residence time in Delhez et al. (2004), Eq. (3) or Eq. (6) must be solved with homogenous boundary conditions prescribed on the boundary $\delta \omega$ of the control domain $\omega$. In particular, $\bar{\theta}$ must vanish at the boundary of the control domain.

With such boundary conditions, water parcels leaving the domain at some time are not allowed to re-enter and $D(t, \tau, \mathrm{x})$, which represents the mass in the control domain at time $t+\tau$ after a unit injection, is a decreasing function of $\tau$. This decreasing behavior is of course expected from the interpretation of $D$ as a cumulative distribution function. It is also required to transform the usual definition of the 

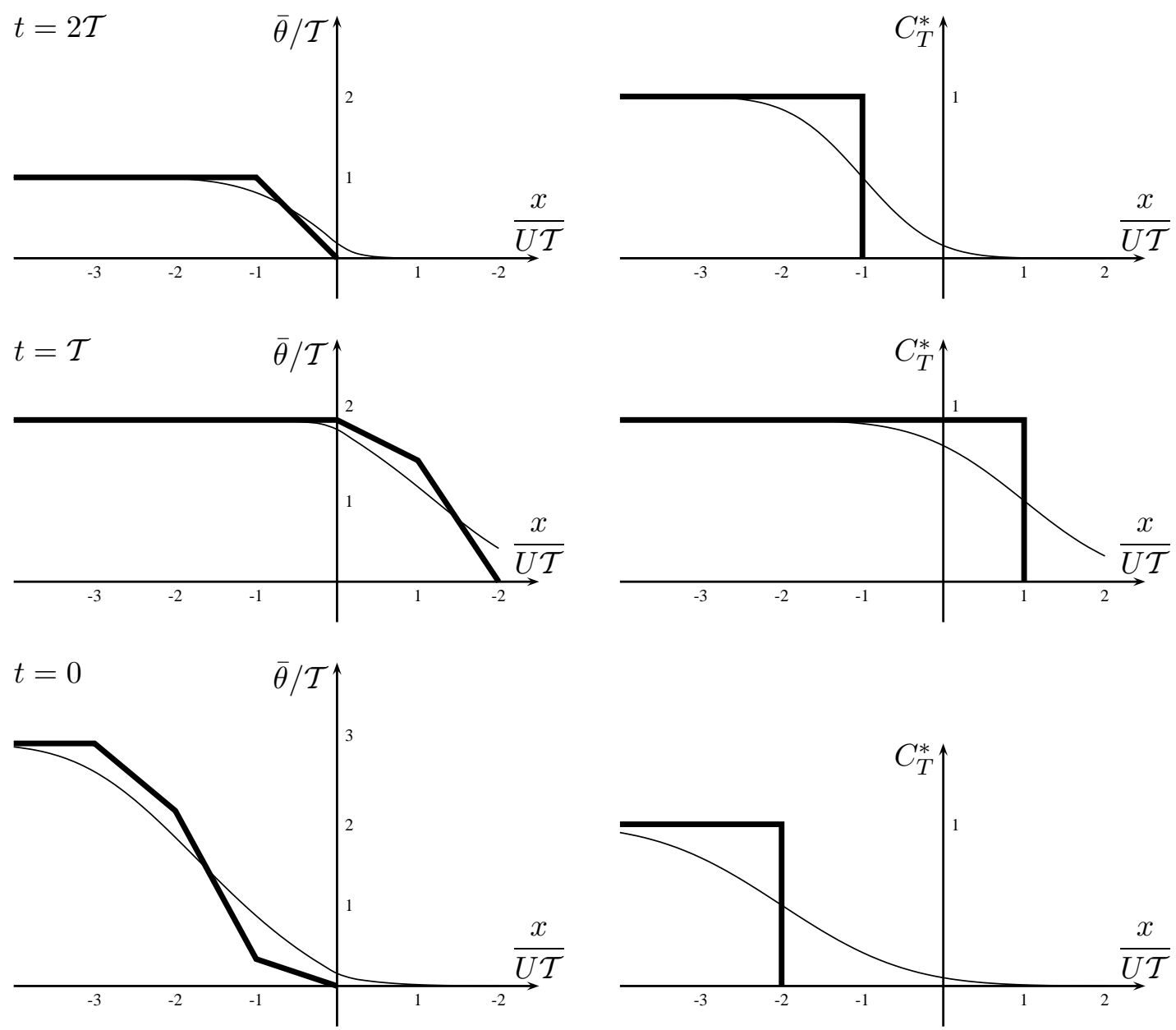

Fig. 3. Temporal evolution of $\bar{\theta}$ and $C_{T}^{*}$ from a backward integration of the equations for exposure time from $t=T$. Results without diffusion (thick curve) and with diffusion (thin curve, $\kappa=U^{2} \mathcal{T} / 4$ ).

residence time (1) into (5) along

$$
\begin{aligned}
\bar{\theta}(t, \mathrm{x}) & =\int_{0}^{1} \tau d \tilde{m}_{(t, \mathrm{x})}=-\int_{0}^{\infty} \tau \frac{d \tilde{m}_{(t, \mathrm{x})}}{d t}(t+\tau) d \tau \\
& =-\left[\tau \tilde{m}_{(t, \mathrm{x})}(t+\tau)\right]_{0}^{\infty}+\int_{0}^{\infty} \tilde{m}_{(t, \mathrm{x})}(t+\tau) d \tau \\
& =\int_{0}^{\infty} \tilde{m}_{(t, \mathrm{x})}(t+\tau) d \tau \\
& =\int_{0}^{\infty} D(t, \tau, \mathrm{x}) d \tau=\bar{\Theta}(t, \mathrm{x})
\end{aligned}
$$

(assuming that $\tilde{m}_{(t, \mathrm{x})}$ decreases to zero for large $\tau$ ).

In Delhez et al. (2004), it is proposed to solve Eqs. (3) or (6) with boundary conditions allowing the water parcels to re-enter in the control domain. In this case, the mass $\tilde{m}_{(t, \mathrm{x})}(t+\tau)$ is no longer a decreasing function of the delay $\tau$. Therefore, the first equality in Eq. (23) is not valid and the solution of Eqs. (3) or (6) cannot be interpreted as the residence time any more. Still $D$ and $\bar{\theta}$ have an interesting interpretation: they can be regarded as measures of the total time spent by the water parcels in the control domain. In particular, $\bar{\theta}$ measures the area under the curve $\tilde{m}_{(t, \mathrm{x})}(t+\tau)$ for the whole range of values of $\tau$ (or $\tau$ in $[0, T-t]$ for finite range simulations). We propose therefore to call this quantity "exposure time".

The concept of exposure time and its computation can also be demonstrated with the idealized one-dimensional system introduced above. This time, Eqs. (6) and (8) must be solved in the whole spatial domain $x \in(-\infty, \infty)$ without prescribing auxiliary conditions at the boundary $x=0$ of the control domain. The results are shown in Fig. 3.

At all times and locations, the value reported for $\bar{\theta}$ measures the total time spent by the water parcels in the control domain between the current time $t$ and the initialization time $T$. The concentration $C_{T}^{*}$ of the control scalar can be used to identify the water parcels that are still in the control domain at $t=T$ and those which left (and did not re-enter) the domain before that time. 


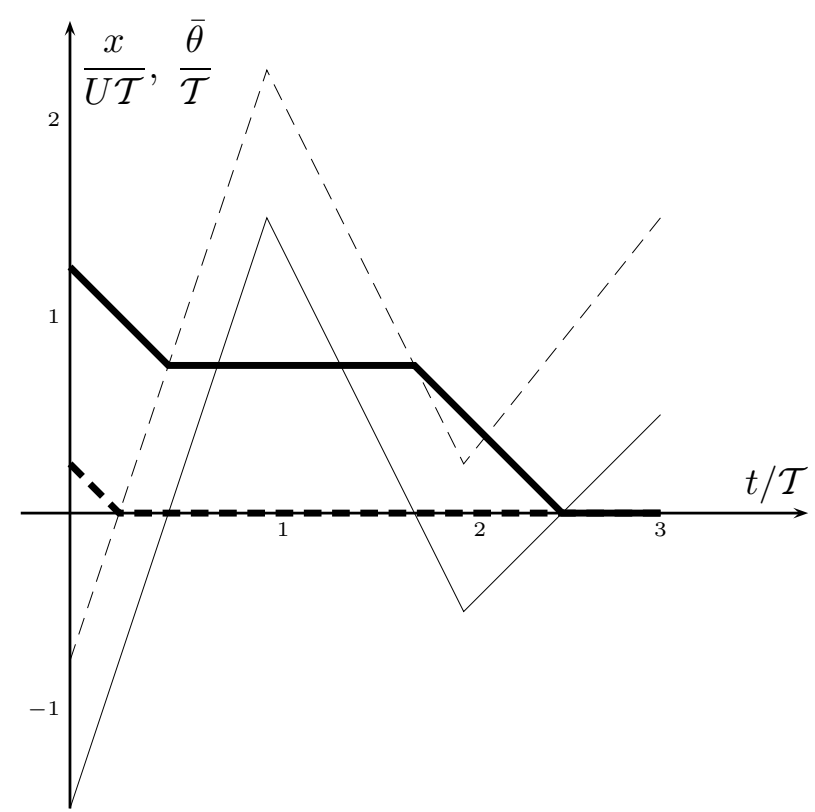

Fig. 4. Temporal evolution of the location (thin curve) and exposure time (thick curve) of particles released at $x=-1.5 U \mathcal{T}$ (plain curve) and $x=-0.75 U \mathcal{T}$ (dashed curve) at time $t=0$.

At $t=2 \mathcal{T}$, the spatial distributions of $\bar{\theta}$ and $C_{T}^{*}$ are identical to those computed in the previous section. This is of course due to the fact that the velocity is positive for $t \in(2 \mathcal{T}, 3 \mathcal{T})$, i.e. that all the water parcels are leaving the control domain.

Between $t=2 \mathcal{T}$ and $t=\mathcal{T}$, all the water parcels that left the domain are advected back into the control domain by the reversing flow (Fig. 1). The value of $\bar{\theta}$ plotted in the left panel for $t=\mathcal{T}$ is affected by the initialization at $t=T$ in the range $x<U \mathcal{T}$ as shown by the value of $C_{T}^{\star}$ in this part of the domain. The results for $x>U \mathcal{T}$ are not affected by the initialization. The values reported for $\bar{\theta}$ in this range can therefore be understood as the true exposure time of the water parcels, i.e. as a measure of the time spent by the water parcels in the control domain. This measure is representative inasmuch as the water parcels have left the control domain before $t=T$. Of course, a reversal of the flow at $t>T$ could however push the parcels back into the control domain at later times.

Particles in $x<-2 U \mathcal{T}$ at $t=0$ are still in the control domain at $t=T$ while those located at $x>-2 U \mathcal{T}$ at time $t=0$ have all left the control domain at $t=T$. The latter exhibit exposure times between 0 and $2 \mathcal{T}$. The stair-case distribution of $\bar{\theta}$ reflects the different paths of the parcels. As shown in Fig. 4 in the particular case of particles released at $x=-1.5 U \mathcal{T}$ and $x=-0.75 U \mathcal{T}$, some particles are present in the control domain during two distinct time intervals while others spent their time in $\omega$ in one single time interval. In both cases, the exposure time is the accumulated time spent in the control domain.

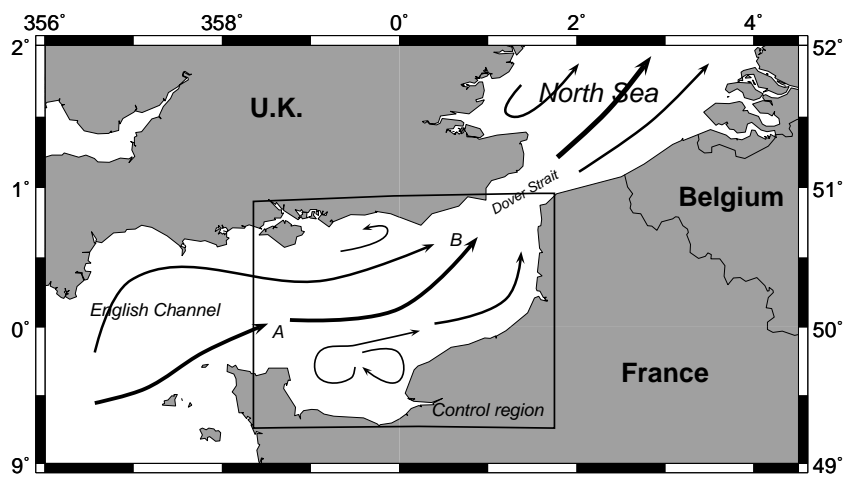

Fig. 5. Schematic view of the general circulation in the English Channel and location of stations A and B.

Similar results are obtained when diffusion is added to the system (Fig. 3). As for the residence time, the spatial distribution are smoother and the effect of the initialization is increased by diffusion. This last effect appears even worse in Fig. 3 than in Fig. 2. There is indeed no strong boundary condition at $x=0$ which can constrain the solution and make it converge faster.

\section{Realistic application}

The concepts introduced above are illustrated here with results of realistic simulations carried out with a threedimensional hydrodynamic model of the Northwestern European Continental Shelf (Delhez and Martin, 1992). The model domain covers the whole shelf between $48^{\circ} \mathrm{N}$ and $62^{\circ} \mathrm{N}$. The shelf break ( $200 \mathrm{~m}$ isobath) is the Western boundary. The model horizontal resolution is $10^{\prime} \times 10^{\prime}$ in longitude and latitude. Sigma coordinates, with 10 levels, are used in the vertical.

A schematic description of the residual circulation in this region is shown in Fig. 5. In addition to this picture, the region is known for its strong tidal signal with a characteristic tidal velocity of about $1 \mathrm{~m} / \mathrm{s}$.

For this illustration, the Eastern Basin of the English Channel is taken as control domain (Fig. 5). The residence time and exposure time are computed from the results of (backward) simulations running from September 1995 to July 1993. Realistic 6 hourly wind forcing data (NCEPreanalysis) are used to force the model.

The Figs. 6 and 7 show time series of the residence time and exposure time and related control scalar concentrations at the surface at two stations A and B in the control domain (see Fig. 5 for the location of these stations). Snapshots of the spatial distribution of the residence time can be found in Delhez et al. (2004).

In both Figs. 6 and 7, the concentration of the control scalar is seen to decrease with a time scale that is of the same 


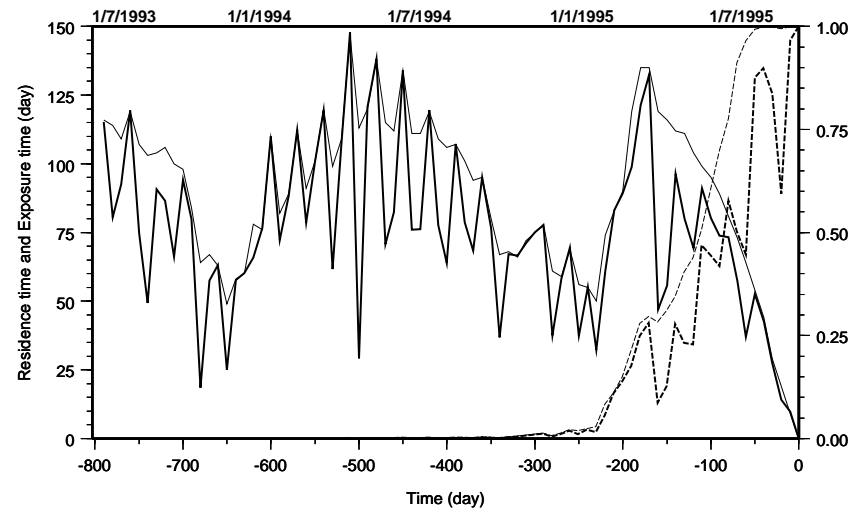

Fig. 6. Time series of the residence time (thick solid curve) and exposure time (light solid curve) at station A (see Fig. 5 for location). The corresponding time series for the concentration of the control scalar (right axis) are plotted with dotted lines (thick curve - residence time/light curve $=$ exposure time).

order of magnitude as the mean residence/exposure time. The control scalar for the residence time decreases slightly more rapidly than its counterpart for the exposure time. The potential biais introduced by the initialisation procedure can be neglected after about 220 days at station A and after about 40 days at station $B$.

The time average values of the residence times at stations $\mathrm{A}$ and $\mathrm{B}$ are, respectively, around 85 and 7 days. As expected from the definition of the two concepts, the exposure time is larger than the residence time at all times and locations.

The differences between the two concepts are small at station B which is located close to the downstream boundary of the control region. At station A, on the contrary, large differences are computed. The residence time at this location shows large temporal oscillations which do not appear in the time series of the exposure time. These oscillations are induced by episodes during which the influence at station $\mathrm{A}$ of the western boundary of the control domain increases (These events are poorly sampled by the 10 day model outputs and will be the subject of further investigations).

\section{Conclusion}

The method introduced by Delhez et al. (2004) provides a versatile tool to diagnose complex flows. In this paper, we showed how the issues of boundary conditions and initial conditions must be approached.

According to the kind of boundary conditions that are applied to the numerical tracer, the method delivers the true residence time or the exposure time. Both concepts provide interesting information about the combined effects of advection and diffusion and are useful in different contexts. Awareness about the differences of the two concepts is essential for

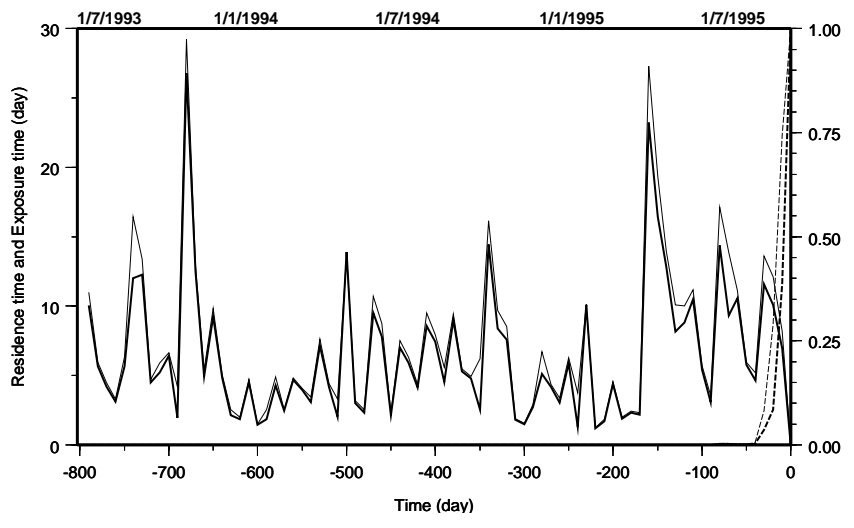

Fig. 7. Time series of the residence time (thick solid curve) and exposure time (light solid curve) at station B (see Fig. 5 for location). The corresponding time series for the concentration of the control scalar (right axis) are plotted with dotted lines (thick curve - residence time/light curve $=$ exposure time).

the correct interpretation of these diagnostics. The true residence time in a control domain $\omega$ measures the (average) time spent by water/tracer parcels in $\omega$ until they leave this control domain. The newly introduced exposure time measures the accumulated time during which a control region is affected by a pollutant released in this region, even if the presence of the pollutant in $\omega$ is intermittent.

Both academic and realistic examples demonstrate the different dynamics of these two diagnostics.

By resorting to the computation of a control scalar, the effect of the initialization on the computed residence/exposure times can be assessed. The concentration of the control scalar must be as small as possible to avoid any bias of the results by the initialization procedure.

Acknowledgements. The author thanks E. Deleersnijder for useful suggestions. This is MARE publication no. 75 .

Edited by: J. M. Huthnance

\section{References}

Bolin, B. and Rhode, H.: A note on the concepts of age distribution and residence time in natural reservoirs, Tellus, 25, 58-62, 1973.

Braunschweig, F., Martins, F., Chambel, P., and Neves, R.: A methodology to estimate renewal time scales in estuaries: the Tagus Estuary case, Ocean Dynamics, 53(3), 137-145, 2003.

Deleersnijder, E., Campin, J.-M., and Delhez, E. J. M.: The concept of age in marine modelling: I. Theory and preliminary model results, J. Marine Syst., 28, 229-267, 2001.

Delhez, E. J. M. and Martin, G.: Preliminary results of 3-D baroclinic numerical models of the mesoscale and macroscale circulations on the North-Western Europena Continental Shelf, J. Marine Syst., 3(4-5), 423-440, 1992. 
Delhez, E. J. M., Campin, J.-M., Hirst, A. C., and Deleersnijder, E.: Toward a general theory of the age in ocean modelling, Ocean Modelling, 1, 17-27, 1999.

Delhez, E. J. M. and Deleersnijder, E.: The concept of age in marine modelling: II. Concentration distribution function in the English Channel and the North Sea, J. Marine Syst., 31, 279-297, 2002.

Delhez, E. J. M., Heemink, A. W., and Deleersnijder, E.: Residence time in a semi-enclosed domain from the solution of an adjoint problem. Estuarine, Coastal and Shelf Science, 61, 691702, 2004.

Harley, S. J., Myers, R. A., and Field, C. A.: Hierarchical models improve abundance estimates: Spawning biomass of hoki in Cook Strait, New Zealand, Ecological Applications, 14/5, 14791494, 2004.

Hydes, D. J., Gowen, R. J., Holliday, N. P., Shammon, T., and Mills, D.: External and internal control of winter concentrations of nutrients (N, P and $\mathrm{Si}$ ) in north-west European shelf seas, Estuarine, Coastal and Shelf Science, 59/1, 151-161, 2004.

Monsen, N. E., Cloern, J. E., and Lucas, L. V.: A comment on the use of flushing time, residence time and age as transport time scales, Limnology and Oceanography, 47(5), 1545-1553, 2003.
Nixon, S. W., Ammerman, J. W., Atkinson, L. P., Berounsky, V. M., Billen, G., Boicourt, W. C., Boynton, W. R., Church, T. M., Ditoro, D. M., Elmgren, R., Garber, J. H., Giblin, A. E., Jahnke, R. A., and Owens, N. J. P.: The fate of nitrogen and phosphorus at the land-sea margin of the North Atlantic Ocean, Biogeochem., 35, 141-180, 1996.

Takeoka, H.: Fundamental concepts of exchange and transport time scales in a coastal sea, Continental Shelf Research, 3, 311-326, 1984.

Vollenweider, R. A.: Advances in defining critical loading levels of phosphorus in lake eutrophicatio, Mem. Ist. Ital. Idrobiol. 33, 53-83, 1976.

Wang, J. D., Luo, J., and Ault, J. S.: Flows, salinity, and some implications for larval transport in South Biscayne Bay, Florida, Bull. Marine Sci., 72/3, 695-723, 2003.

Zimmerman, J. T. F.: Estuarine residence times, in: Hydrodynamics of estuaries, edited by: Kjerfve, B., Hydrodynamics of estuaries, CRC Press, 1, 75-84, 1988. 